\title{
Encapsulación de Aditivos para la Industria de Alimentos
}

* Ingeniera Química - Aspirante a Doctor en Ingeniería - Área Ingeniería de Alimentos - Facultad de Ingeniería Universidad del Valle, Santiago de Cali, Colombia. E-mail:angelasan@univalle.edu.co

* * Ingeniero Químico - Aspirante a Doctor en Ingeniería - Área Ingeniería de Alimentos - Facultad de Ingeniería Universidad del Valle, Santiago de Cali, Colombia. E-mail:edurosa@univalle.edu.co

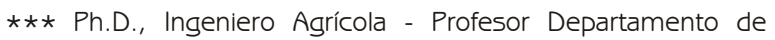
Ingeniería de Alimentos - Facultad de Ingeniería Universidad del Valle, Santiago de Cali, Colombia. E-mail:alfayala@univalle.edu.co

Fecha de recepción: Moviembre 20 de 2003 Fecha de aprobación: Abril 30 de 2004

\author{
Angélica Sandoval Aldana* \\ Eduardo Rodríguez Sandova** \\ Alfredo Ayala Aponte***
}

\section{RESUMEN}

La encapsulación es una técnica que se emplea para proteger los aditivos utilizados en la industria de alimentos, Esta técnica tiene diferentes propósitos en un producto alimenticio, entre los que se destacan: la conservación, fortificación y liberación controlada en el tiempo de nutrientes, disminución de la higroscopicidad, transformación de sabores líquidos a polvos, estabilidad durante el almacenamiento, mejoramiento de cualidades organolépticas y funcionales de productos alimenticios, etc. Los principales aditivos encapsulados en la industria de alimentos son: ácidos, colorantes, pigmentos, enzimas, microorganismos, sabores, especies, 
grasas y aceites, vitaminas, minerales, sales, edulcorantes, gases y agentes leudantes. La selección del material de recubrimiento es el paso más importante para obtener un producto encapsulado porque dependiendo de sus propiedades, se puede cumplir con los requerimientos de protección. El método de encapsulación se elige de acuerdo a la aplicación requerida, el tamaño de cápsula deseado, el material a encapsular, el costo y las propiedades físicas y químicas del recubrimiento. Las investigaciones en torno a este tema se centran en la búsqueda y aplicación de nuevos materiales de recubrimiento y técnicas novedosas de encapsulación. Este artículo es una revisión de la técnica de encapsulación, su fundamento, los métodos más utilizados en la industria de alimentos y algunos estudios sobre diferentes materiales de recubrimiento.

Palabras claves: encapsulación, métodos de encapsulación, aditivos para alimentos, materiales de recubrimiento.

\section{ABSTRACT}

Encapsulation is a technique that is applied to protect additives or ingredients in food industry. This technique provide different qualities to food products such as conservation, fortification and controlled the release of nutrient, decreasing the hygroscopicity, flavor transformation from liquid to powder form, storage stability, improved sensory and functional properties of food products, etc. The main encapsulated additives in food industry are: flavor, acids, vitamins, minerals, species, micro-organisms, colorants, enzymes, salts, savory, fats, gases and bakery agents. The choice of coating material is the most important step to obtain an encapsulated product because the protection requirements depend on its properties. The encapsulation method is chosen according to the application, the capsulate size, the material to encapsulate, the cost, and the physic- chemical properties of coating. Studies are aimed at searching and applying new coating materials and encapsulation techniques. This article presents the concepts of encapsulation, the techniques most applied in food industry, and some researches about different coating materials.

Key words: encapsulation, food additives, coating materials, encapsulation techniques

\section{INTRODUCCIÓN}

La encapsulación de aditivos para la industria de alimentos es una técnica antigua; sin embargo, cada día encuentra mayor aplicación en resolver problemas para la conservación de productos alimenticios al mantener su calidad organoléptica. Los aditivos encapsulados presentan mayor estabilidad a factores ambientales y limitan su interacción con otros ingredientes, asegurando los distintos efectos que se requieren. En el caso de aditivos funcionales para el organismo, como minerales, vitaminas y probióticos, la encapsulación otorga un mayor beneficio asegurando que su liberación sea controlada.

La técnica de encapsulación de aditivos se considera como un factor de competitividad entre industrias ya que permite ofrecer al consumidor un producto con mejores cualidades organolépticas y funcionales. En la Tabla 1, se presentan los principales centros de investigación sobre esta técnica en el mundo.

Este trabajo presenta una revisión de la técnica de encapsulación aplicada en la industria de alimentos, su fundamento, los métodos comúnmente utilizados y algunos estudios recientes sobre diferentes materiales de recubrimiento.

\section{GENERALIDADES}

La encapsulación se considera como un empaque especial en el cual un recubrimiento individual se aplica a partículas sólidas pequeñas, gotas de líquido o gases, protegiéndolas contra factores ambientales perjudiciales $[1,2]$. El recubrimiento es semipermeable, protege al centro activo de condiciones severas y controla el 
Tabla 1. Centros de Investigación sobre encapsulación de aditivos alimenticios

\begin{tabular}{|c|c|c|c|}
\hline Instituto & Investigador & Clase & Áreas de Investigación \\
\hline EMITIAA (Francia) & Dr. Poncelet & Escuela de Ingeniería & Ingeniería de la Encapsulación \\
\hline ATO B.V (Holanda) & M.H. Vingerhoeds & Centro de Investigación & $\begin{array}{l}\text { Aplicaciones en Procesos de } \\
\text { Alimentos, Extrusión }\end{array}$ \\
\hline University of Birmingham (UK) & Dr. Z. Zhang & Centro de Investigación & $\begin{array}{l}\text { Formulación de Micricápsulas } \\
\text { y su Caracterización }\end{array}$ \\
\hline VTT Biotchnol. (Finlandia) & Dr. P. Forssell & Centro de Investigación & $\begin{array}{l}\text { Matrices de Encapsulación de } \\
\text { Base de Almidón }\end{array}$ \\
\hline UPVIEHU LEIA Fundation (España) & Prof. J.L. Pedraz & Fundac. de Investigación & Aplicaciones en Alimentos \\
\hline GeniaLab Bio Technologie (Alemania) & Dr. Ulrich Jahnz & Centro de Investigación & $\begin{array}{l}\text { Tecnología y Materiales de } \\
\text { Encapsulación }\end{array}$ \\
\hline CERTH/CPERIMPRE (Grecia) & Prof. Kostas Kiparissides & Instituto de Investigación & Liberación Controlada de Aditivos \\
\hline ITZ - Bremerhaven (Alemenia) & Dr. C. Maehr & $\begin{array}{l}\text { Centro de Transferencia } \\
\text { de Tecnología }\end{array}$ & $\begin{array}{l}\text { Diseño de Producto y de Procesos, } \\
\text { Secado por Aspersión }\end{array}$ \\
\hline Università di Perugia (Italia) & Prof. C. Mastruzzi & Unversidad & Liberación Controlada \\
\hline $\begin{array}{l}\text { Food reserarch and development } \\
\text { center (Canadá) }\end{array}$ & Ph.D. Edward Fainworth & Centro de Investigación & Mutraceuticos, alimentos saludables \\
\hline $\begin{array}{l}\text { Memorial University of } \\
\text { Mewfoundland (Canadá) }\end{array}$ & Dr. Shahidi Fereidoon & Universidad & $\begin{array}{l}\text { Aplicaciones en Productos } \\
\text { Comerciales }\end{array}$ \\
\hline Southwest Research Institute (USA) & Ph.D. Miraj Vasishtha & Centro de Investigación & Muevas Técnicas, Extrusión \\
\hline $\begin{array}{l}\text { Eastern regional Research Center } \\
\text { (USA) }\end{array}$ & Dr. Charles Onwulata & Centro de Investigación & $\begin{array}{l}\text { Aplicación de Productos derivados } \\
\text { lácteos }\end{array}$ \\
\hline University of Rutgers (USA) & Ph.D. King & Universidad & Aplicación con Hidrocoloides \\
\hline University of Minnesota & Dr. G.A. Reineccius & $\begin{array}{l}\text { Departamento de Ciencia } \\
\text { Alimentos y Mutrición }\end{array}$ & $\begin{array}{l}\text { Encapsulación de sabores, } \\
\text { principalmente técnica de secado } \\
\text { por aspersión }\end{array}$ \\
\hline
\end{tabular}

flujo de sustancias [3]. El material protegido se conoce como corazón, centro activo, relleno o fase interna. El material protector se llama cápsula, recubrimiento, pared, membrana o matriz [4].

Los términos microencapsulación y encapsulación se han utilizado indistintamente para describir el proceso de proteger un centro activo con un recubrimiento, la diferencia entre los términos se basa en el tamaño de la cápsula $[1,5,6]$. El recubrimiento en la encapsulación puede ser: simple o de múltiples centros, pared de forma regular esférica, pared irregular, estructura multicompuesta del mismo material o de diferentes materiales $[4,6]$.

El estudio de la encapsulación comenzó en los años 1930 's, por B.K. Green, quien utilizó sus estudios sobre materiales coloidales para el desarrollo de las copias sin papel carbón [5]. El método más antiguo utilizado en alimentos es el secado por aspersión, el cual comenzó encapsulando sabores en goma arábiga a finales de la década de los años 1930's [5,7]. En los años 1950 's, Swisher, patentó un método para encapsular aceites esenciales vía extrusión [8]. Otro pionero en la investigación fue el Instituto de 
Investigaciones de SouthWest [USA], el cual durante la década de los años 60 's, trabajó en la encapsulación de aceites esenciales para prevenir la oxidación y pérdida de compuestos volátiles [9].

La industria farmacéutica colaboró ampliamente en el desarrollo de nuevas técnicas de microencapsulación. La técnica de lecho fluidizado, desarrollada por D.E. Wuster en los años 1950's, se ha utilizado para producir el recubrimiento de las drogas [10], el cual asegura una dosificación apropiada, mejora la estabilidad del ingrediente activo, controla la liberación y enmascara el sabor $[1,2]$.Otras industrias donde actualmente se aplica la encapsulación son la cosmética y la agrícola, en esta última se encapsulan pesticidas y fertilizantes garantizando su liberación constante durante un tiempo determinado [11].

\subsection{Objetivos de la encapsulación de aditivos en la industria de alimentos}

La industria de alimentos utiliza la técnica de encapsulación por diferentes razones $[1,5,7,12]$ :

■ Disminuir la velocidad de evaporación o de transferencia del material central hacia el medio ambiente externo.

घ Controlar la liberación del material central a condiciones predeterminadas como cambio de $\mathrm{pH}$ o humedad, aplicación de calor o estímulos físicos.

$\square \quad$ Reducir la interacción entre el material central y el ambiente externo: algunos ingredientes son sensibles al calor, la luz y la humedad y otros son altamente reactivos y tienden a oxidarse $y$ volatilizarse.

■ Facilitar la manipulación del material central. La encapsulación convierte un líquido a estado sólido, además previene la agregación, favorece el proceso de mezclado y asegura que el material central se encuentre uniforme en la mezcla.

v Enmascarar el sabor del material central.

\subsection{Material de recubrimiento}

El paso inicial en la encapsulación es la elección del material de recubrimiento apropiado. Aunque el método de encapsulación puede afectar el mecanismo de liberación del centro activo, la formulación del recubrimiento por sí mismo es el factor más determinante [7]. El material protector debe reunir ciertas propiedades que dependen de las características químicas del material encapsulado, aplicación, condiciones de almacenamiento y proceso al cual será expuesto [2].

Las características de un recubrimiento ideal para encapsular son $[2,7,6]$ :

Baja viscosidad a altas concentraciones.
Baja higroscopicidad para facilitar su
manipulación y evitar la aglomeración.
Capacidad de emulsificar y estabilizar el
material central.
Insoluble y no reactivo con el material
central. El recubrimiento es soluble en los
solventes alimenticios comunes, o en el
Producto alimenticio final.
Proporcionar máxima protección al material
central contra condiciones adversas como
la luz, el pH, el oxigeno, la humedad y otros
ingredientes reactivos.
Permitir la liberación completa de solventes
y otros materiales usados durante el
proceso de encapsulación.
$\square \quad$ Sabor insípido
Poseer bajo costo

Los materiales de recubrimiento se seleccionan de una amplia variedad de polímeros sintéticos y naturales [Tabla 2], estos materiales se pueden mezclar entre ellos para obtener propiedades de barrera y mecanismo de liberación específicos; o se puede combinar con modificadores como antioxidantes o surfactantes [7].

\subsection{Mecanismos de liberación del centro activo}

El material de recubrimiento debe ofrecer algún mecanismo de liberación del centro activo a un tiempo determinado. Los principales 
Tabla 2. Clases de Material de Recubrimiento

\begin{tabular}{|l|l|}
\hline \multicolumn{1}{|c|}{ Clases de Material de Recubrimiento } & \multicolumn{1}{c|}{ Tipos Específicos de Recubrimiento } \\
\hline Gomas & Goma arábiga, agar, alginato de sodio, carrangenina \\
\hline Carbohidratos & Almidón, Maltodextrinas, sacarosa, jarabe de maíz, ciclodextrinas \\
\hline Celulosas & $\begin{array}{l}\text { Carboximetil celulosa, metil celulosa, etil celulosa, nitrocelulosa, } \\
\text { acetilcelulosa }\end{array}$ \\
\hline Lípidos & $\begin{array}{l}\text { Cera, parafina, triestarina, ácido esteárico, monogliceridos, digliceridos, } \\
\text { cera de abejas, aceites, grasas }\end{array}$ \\
\hline Materiales inorgánicos & Sulfato de calcio, Silicato \\
\hline Proteínas & Gluteína, caseína, gelatina, albúmina \\
\hline
\end{tabular}

mecanismos de liberación que se aplican en el sector de alimentos son los siguientes:

■ Disolución o fusión: La integridad de la cápsula se destruye por disolución en un solvente apropiado o por acción del calor. Los recubrimientos hidrosolubles se disuelven fácilmente con el incremento de la humedad, la adición de agentes químicos o ajustándose a diferentes niveles de sales [7]. La liberación térmica se utiliza en cápsulas con material protector de base grasa, el cual funde y libera el centro activo [12].

■ Liberación física: El material protector se fractura por fuerzas externas como presión o fricción. La masticación es el principal mecanismo de liberación, igualmente, durante la mezcla de las materias primas se presenta la liberación por fricción [12].

$\square$ Difusión: este proceso de liberación es guiado por el gradiente de concentración y las fuerzas atractivas entre cadenas, como puentes de hidrógeno, fuerzas de Van der Waals, grado de entrecruzamiento y cristalinidad [7]. Además, está controlado por la solubilidad y la permeabilidad del material central en el material protector [6]. En los métodos físicos de encapsulación se busca la formación de una estructura amorfa metaestable, de baja permeabilidad al compuesto encapsulado, al oxígeno y otros compuestos, y con alta temperatura de transición vítrea [7]. La permeabilidad del material protector cambia al someterse a condiciones específicas de temperatura y humedad. Los principios físico-químicos de la transición vítrea de estos materiales han sido estudiados por Levine et al., concluyendo que la liberación del centro activo se presenta en la transición del estado vítreo a estado gomoso, por calentamiento de la matriz. El material central se libera por difusión a una velocidad que aumenta con el incremento de la temperatura. La estabilidad de cápsula depende de que su temperatura de transición sea superior a la temperatura de almacenamiento [13].

\section{MÉTODOS DE ENCAPSULACIÓN}

La selección del método a encapsular depende de los costos, el tamaño de la cápsula, las propiedades físicas y químicas de los materiales, la aplicación y el mecanismo de liberación deseado. Existen muchos métodos que son propiedad intelectual de las empresas $y$, por lo tanto, su conocimiento es restringido [12]. Los siguientes métodos son los de mayor aplicación en la industria de alimentos $[6,7]$ :

$\square$ Métodos físicos: secado por aspersión, enfriamiento por aspersión, liofilización, recubrimiento por lecho fluidizado, extrusión, co-extrusión, extrusión-fusión, cocristalización. 
ఐ Métodos físico-químicos: coalescencia, inclusión molecular, encapsulación por liposomas.

\subsection{Métodos Físicos}

Secado por aspersión: es el método de mayor uso y el de menor costo (Figura 1). El proceso requiere tres pasos básicos, la formación de la emulsión entre el material central y el recubrimiento, la homogenización y la aspersión. La emulsión se atomiza dentro de una corriente de aire caliente, al evaporarse el agua los sólidos remanentes forman una cápsula alrededor del material central por atracción másica, la remoción rápida del agua mantiene la temperatura del centro por debajo de $100^{\circ} \mathrm{C}$. El tamaño promedio de la cápsula obtenida es 100 $\mathrm{mm}$ y su recolección se realiza mediante ciclones. Las propiedades físicas de las microcápsulas dependen de la temperatura del aire caliente, el grado de uniformidad de la aspersión y el contenido de sólidos en la emulsión $[2,3,7]$.

\section{Enfriamiento y descenso de la temperatura por} aspersión: estos métodos son la antítesis del secado por aspersión, aquí el recubrimiento es una cera o grasa y las partículas se forman a través del enfriamiento. Las cápsulas protegen el centro activo de la humedad y la liberación se realiza a la temperatura de fusión del recubrimiento [12]. La diferencia en los puntos de fusión del material protector denomina cada método, en el enfriamiento se utilizan lípidos, como ácido esteárico y mono- y di-glicéridos con puntos de fusión entre $45-122^{\circ} \mathrm{C}$, en el segundo método aceites fraccionados o hidrogenados [p.f: $\left.32-45^{\circ} \mathrm{C}\right][2,4]$.

Liofilización: este proceso de deshidratación se realiza a temperaturas y presiones bajas garantizando así alta retención de compuestos volátiles. Es un método simple y suave pero con un largo período de deshidratación [2], además las cápsulas presentan una estructura porosa que facilita la interacción con el medio ambiente [14].
Se utiliza para la encapsulación de esencias solubles en agua, aromas naturales y drogas [7].

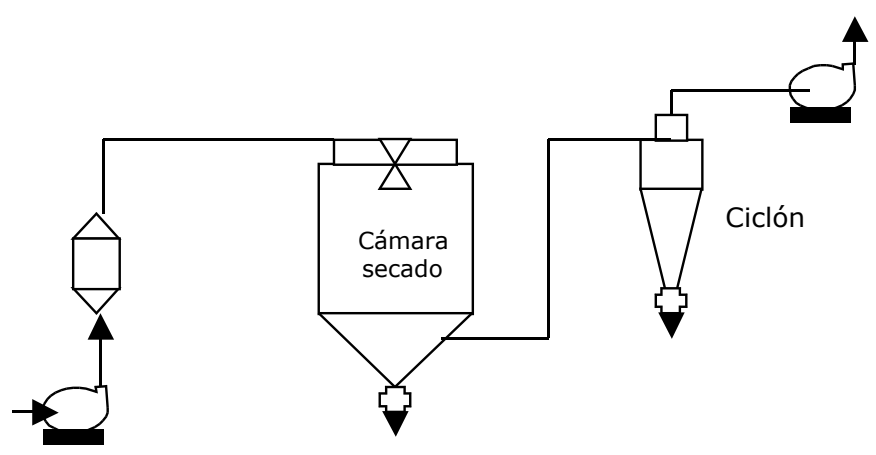

Figura 1. Diseño típico de un secador por aspersión

Recubrimiento en lecho fluidizado: esta técnica es útil cuando el material central son partículas sólidas, las cuales se localizan en una cámara con corriente de aire hacia arriba donde el recubrimiento se atomiza $[2,4,12]$. Las partículas hacen ciclos aleatorios dentro de la cámara con el fin de recibir sucesivas capas delgadas, lo que posibilita la aplicación de diferentes tipos de material de recubrimiento. Sin embargo, la aspersión La aspersión es el de mayor aplicación en la industria de alimentos porque ofrece mayor versatilidad y altos volúmenes de producción $[7,10]$.

Extrusión y Co-extrusión: la extrusión consiste en forzar a través de moldes la masa fundida del recubrimiento con el material central dispersó, la cápsula se forma por endurecimiento del material protector al entrar en contacto con un líquido deshidratante [7]. El proceso patentado por Swisher, requería la formación de una emulsión, la cual se extruía en pellets o se sometía a agitación dentro de un líquido caliente inmiscible. Las cápsulas se formaban por choque térmico al enfriarse rápidamente [8]. En el Instituto Southwest Research [U.S.A], estudiaron y desarrollaron los métodos de co-extrusión en el principio de tubos concéntricos, donde el material central fluye en el tubo interno y el recubrimiento en el externo $[7,12,15]$. 
Extrusión - fusión: en este proceso se requiere mezclar distintos materiales de recubrimiento para obtener una matriz vítrea estable a temperatura de almacenamiento, asegurando así que la difusión de los compuestos volátiles se realice a condiciones determinadas de humedad y temperatura. La matriz vítrea no es porosa lo que asegura que no hay interacción entre el oxigeno y el material central. Se utiliza un extrusor de tornillo simple o doble, el cual funde y forma el material de recubrimiento y el central en un proceso continuo, se debe mantener un contenido de agua mínimo para garantizar una temperatura de transición vítrea alta. Al aumentar la humedad se libera el material encapsulado [13, 16].

Co-cristalización: es la inclusión de compuestos dentro de agregados de sacarosa, al cambiar la estructura perfecta del cristal a un conglomerado, el cual admite la adición de un segundo ingrediente. La cápsula obtenida es granular, de fácil manejo y con buenas características de flujo. El paso inicial para la encapsulación es la concentración de los jarabes de sacarosa hasta su supersaturación, posteriormente se adiciona el material central y se agita constantemente para inducir la nucleación y la aglomeración. La encapsulación de sabores por este método es una alternativa económica y flexible, sin embargo, requiere la adición de un antioxidante que prolongue el tiempo de vida útil $[2,17]$.

\subsection{Métodos Fisicoquímicos}

Coalescencia: es el único método químico que se aplica en alimentos. Consiste en disolver una proteína gelificante y formar una emulsión con el material central. Una vez que la emulsión está lista, la coalescencia se inicia de diferentes formas como cambio de temperatura, $\mathrm{pH}$ o adición de sustancias (sales iónicas). En este punto la proteína se aglomera adhiriéndose al aceite, formando pequeñas partículas que empiezan a precipitarse $[4,5,12]$. Las cápsulas se recuperan por filtración o centrifugación, los pasos posteriores pueden ser un secado por aspersión, o proporcionar mayor rigidez a la cápsula adicionando un aldehído que entrecruza las moléculas [1].

Inclusión molecular: es una técnica de encapsulación a nivel molecular y utiliza la betaciclodextrina como agente encapsulante. Debido a su estructura molecular cíclica (Figura 2), la beta-ciclodextrina posee un centro hidrofóbico, mientras que la superficie exterior es hidrofílica. Los complejos se forman en el centro hidrofóbico y se realizan en medio acuoso para facilitar su precipitación y separación; finalmente se seca por métodos convencionales. El uso de ciclodextrinas en alimentos sólo es aprobado en Japón y Europa del Este $[4,7]$.

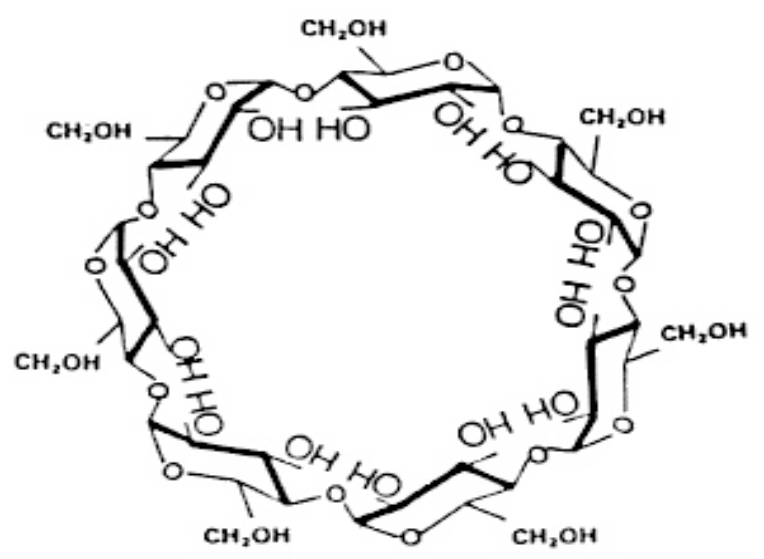

Figura 2. Estructura molecular de la ciclodextrina

Encapsulación por liposomas: Ios liposomas son vesículas formadas por capas de fosfolípidos. El enrollamiento de la capa lipídica en forma esférica forma una cápsula estable debido a que no existe interacción de los lípidos con el agua. Su tamaño varía de pocos nanómetros hasta micrones. Este tipo de cápsula ha sido utilizado en la liberación de vacunas, hormonas, enzimas y vitaminas, en la industria farmacéutica inicialmente, y luego se aplicó en la industria de cosméticos. La ventaja de esta técnica es la facilidad como se cambia la permeabilidad, estabilidad, actividad superficial y afinidad de los liposomas, variando la composición y el tamaño de los lípidos de la membrana. En el almacenamiento se debe evitar 
la exposición al oxigeno y limitar las temperaturas excesivas. Además requieren de la adición de antioxidante $[2,4]$.

\section{INVESTIGACIONES RECIENTES EN MATERIALES DE RECUBRIMIENTO}

Los materiales protectores de mayor uso en la industria de sabores son los carbohidratos. Reinnecius establece las debilidades y fortalezas de las maltodextrinas, los sólidos de jarabe de maíz, almidón modificado y goma acacia, en el secado por aspersión. Las maltodextrinas y los sólidos de jarabe de maíz se caracterizan por no estabilizar las emulsiones con los sabores, lo que produce una baja retención de los compuestos volátiles. El almidón modificado mejora las propiedades emulsificantes; sin embargo, ofrece baja protección contra la oxidación durante el almacenamiento. La goma acacia posee las mejores características en cuanto a estabilización de la emulsión, retención de volátiles y protección contra la oxidación. El autor recomienda trabajar con mezclas de goma acacia y maltodextrinas para disminuir costos [18].

Onwulata et al., estudiaron la encapsulación de grasa de leche en almidón nativo y modificado, y sacarosa, esta última presentó las mejores cualidades de recubrimiento, pues no presenta oxígeno recluido en la cápsula, ni la formación de grandes gotas de grasa que debilitan la estructura y facilitan la difusión del oxigeno. Las matrices de almidón nativo y modificado se caracterizaron por su alta porosidad [19].

La porosidad de las cápsulas de proteína de suero con distintos porcentajes de grasa deshidratada de leche, secadas por aspersión, fueron estudiadas con la técnica de desplazamiento de gas por Moreau y Rosenberg, sus resultados concluyeron que a mayor concentración de grasa la porosidad aumenta, disminuyendo así la calidad del encapsulado [20]. El trabajo similar de Hogan et al., Concluyó que las buenas características de encapsulación de la proteína de suero, están limitadas por la proporción proteína/material central [21].

Los estudios de Dewenttick et al. se centraron en la tendencia del recubrimiento a aglomerarse durante la encapsulación por lecho fluidizado. Realizaron pruebas con esferas de cristal para garantizar que las observaciones dependían únicamente del recubrimiento. En el primer estudio compararon cuatro gomas: locust beam, carboximetil celulosa, alginato de sodio, kcarragenina, concluyendo que el exceso en la velocidad de aspersión favorece la aglomeración. Las diferencias en la forma como se descubrió para las cuatro gomas se explicaron de acuerdo a las características de higroscopicidad, flujo plástico y transiciones dentro de la matriz. El segundo estudio comparó gelatina y almidón hidrolizado, de igual forma la tendencia a la aglomeración depende de la higroscopicidad del material encapsulante, su capacidad de ligar agua y la viscosidad $[22,23]$.

Los sistemas de múltiple recubrimiento es el último diseño para las cápsulas, el cual busca alcanzar distintos objetivos relacionados con el almacenamiento y la liberación controlada $[5,12]$. En la patente de Levine et al., se eliminan o reducen los problemas de los sabores encapsulados vía secado por aspersión, si se realiza una segunda encapsulación dentro de una matriz que posee una temperatura de transición vítrea por encima de la temperatura de almacenamiento, utilizando el método de extrusión-fusión [24].

La investigación de Onwulata et al., Realizó un segundo recubrimiento a aceite de mantequilla encapsulado en sacarosa vía secado por aspersión para evitar la adherencia y agregación. El segundo material protector fue una mezcla de ceras atomizadas sobre el polvo de la primera encapsulación. Los resultados determinaron que la cobertura con cera disminuye las propiedades de flujo de la cápsula y aumenta la resistencia a la absorción de humedad [25]. El recubrimiento con una grasa de un sabor en polvo, secado por aspersión, para obtener la liberación del centro 
activo por temperatura fue realizado por Cho y Park [26]. La cápsula final presentó un mayor tamaño pero disminuyó en un 33\% la absorción de humedad. Este estudio se basó en patentes de doble encapsulación para obtener mayor estabilidad y duración del sabor y poder edulcorante de aplicación principalmente los chicles.

\section{APLICACIONES EN LA INDUSTRIA DE ALIMENTOS}

Los principales aditivos encapsulados en la industria de alimentos son: ácidos, colorantes, pigmentos, enzimas, microorganismos, sabores, especies, grasas y aceites, vitaminas, minerales, sales, edulcorantes, gases y agentes leudantes $[2,27]$.

Los ácidos ayudan en la conservación y el procesamiento de los alimentos; sin embargo, estas sustancias reaccionan degradando colores y sabores. La encapsulación limita la oxidación, disminuye la higroscopicidad, logra una liberación controlada en el tiempo y permite la adición de mayor cantidad de ácido al producto $[7,27]$.

La principal aplicación es la transformación de sabores líquidos a polvos, los cuales presentan menor oxidación y volatilidad $[2,7,27]$. Los colores naturales encapsulados poseen mayor solubilidad y son más estables al oxígeno [6].

Las vitaminas y minerales se adicionan para fortificar una variedad de alimentos, estos aditivos se encapsulan para reducir sabores desagradables, permitir la liberación controlada de nutrientes en el tiempo, aumentar la estabilidad a condiciones extremas de temperatura y humedad y disminuir las reacciones con otros ingredientes [7].

La encapsulación de grasas facilita su manipulación y mezcla con otros ingredientes no grasos, brindando estabilidad durante el almacenamiento y transporte. Adicionalmente, las cápsulas con alto contenido de grasa pueden actuar como emulsificantes [25]. Los lípidos, en estado líquido, son fácilmente susceptibles a la oxidación durante el procesamiento y el almacenamiento, la encapsulación previene estos inconvenientes [7].

Los sistemas de enzimas encapsuladas utilizadas en la fabricación de quesos aceleran la maduración. La enzima es más estable por estar protegida contra factores ambientales como pH y fuerza iónica, lo que permite el desarrollo acelerado de los sabores en los quesos madurados [2].

La encapsulación permite aplicar los microorganismos probióticos en productos no lácteos. Una tecnología de Balchem Corp. utiliza la encapsulación por lecho fluidizado para recubrir con grasa al microorganismo, protegiéndolo de la humedad y la acidez, permitiendo que el probiótico no sea destruido por los jugos gástricos y se libere en el intestino. [28].

La encapsulación de edulcorantes reduce la higroscopicidad, mejora la fluidez y prolonga la percepción de dulce; además permite su aplicación en productos de panadería por ser más estables al calor. Algunos dulces duros se elaboran con dióxido de carbono atrapado, estos al fundirse en la boca producen una ligera sensación de estallido al liberarse el gas; el dulce se produce con la incorporación del gas a presión de 50 o 100 psi en mezclas de azúcares fundidos [2].

\section{CONCLUSIONES}

La encapsulación es una técnica muy útil para la protección de los aditivos utilizados en la industria de alimentos. Esta Técnica se emplea en un producto alimenticio para la prolongación de su vida útil, la fortificación y liberación controlada de nutrientes, la disminución de la higroscopicidad, la transformación de sabores líquidos a polvos, la estabilización durante el almacenamiento y transporte a condiciones extremas de temperatura y humedad, el mejoramiento de 
temperatura y humedad, el mejoramiento de cualidades organolépticas y funcionales de productos alimenticios, la limitación de su interacción con otros ingredientes, etc.

Esta técnica facilita la producción de alimentos funcionales debido a que el recubrimiento ofrece la protección necesaria para la estabilidad del aditivo fortificante, su liberación en la dosis deseada y en el sitio específico del alimento.

Los materiales de recubrimiento de mayor uso en el proceso de encapsulación de aditivos debido a su bajo costo, son los carbohidratos y las gomas, principalmente almidón modificado y goma arábiga, respectivamente.

La encapsulación de sabores mediante la técnica de secado por aspersión es la principal aplicación en la industria de alimentos.

La tendencia de las investigaciones recientes se centra en la generación de nuevos métodos de encapsulación y en conseguir un material de recubrimiento que garantice las condiciones de protección requeridas para distintos aditivos encapsulados, permitiendo obtener mayores aplicaciones en el sector de alimentos y novedosos productos funcionales.

\section{BIBLIOGRAFÍA}

1. BARBOSA, A., et al. Microcápsulas: uma alternativa viável [en línea]. Biotecnologia Ciência \& Desenvolvimento (16). 2000, Pp. 26-30. $<$ http://www.biotecnologia.com. br/novas.html.>

2. BERISTAIM, C., et al. Encapsulation of Orange Peel Oil by Co-crystallization. Lebensmittel Wissenschaft und Technologie. Vol. XXIX, (7). 1996,pp. 645-647.

3. BERRY, D. Hiding Healthful Ingredients. Food product design. [en linea], 2002 $<$ http://www.foodproductdesign.com/archiv e/2002/0502AP.html
4. CHEM, J., JAME, J. Effectiveness of granular cold-water-soluble starch as a Controlledrelease matrix. Cereal chemistry. Vol. LXXII (3), 1995, pp.265-268.

5. CHO, Y.H., PARK, J. Characteristics of doubleencapsulated flavor powder prepared by secondary fat coating process. Journal of food science. Vol. LXVII (3), 2002, Pp. 968-973.

6. DEWETTIMCK, K., et al. Aglomeration tendency during Top-Spray fluidized bed coating with gums. Lebensmittel Wissenschaft und Technologie. Vol. XXXI (6), 1998, pp. 576584.

7. DEWETTIMCK, K., et al. Aglomeration tendency during Top-Spray fluidized bed coating with gelatin and starch hydrolysate. Lebensmittel Wissenschaft und Technologie. Vol. XXXII (2), 1999, pp. 102-106.

8. DEWETTIMCK, K., HUYGHEBAERT, A. Fluidized bed coating in food technology. Trends in Food Science \& Technology. Vol. X, 1999, pp.163168

9. DEYMOMAZ, C, et al. Spraydrying-Microencapsulation. 1998. Disponible en internet <http://www.wsu.edu/ gmhyde/433_web_ pages/drying-web-pages98/spray-dry/S̄prayDrying-intro.htm>

10.FULGER, C., POPPLEWELL, L.M. FI encapsulation. US PATENT 5951502. 1999. Disponible en internet $<$ http://www.uspto.gov $>$

11. GIBBS, B., et al. Encapsulation in the food industry: a review. International Journal of food science and nutrition (50), 1999, Pp. 213-224.

12. GLICKSMAM, M. FOOD HYDROCOLLOIDS, Vol. 1. CRC Press, Mew York: Boca Raton, 1982, p. 86-89. 
13. HEGEMBART, S. Encapsulated ingredients keep problems covered. Food product design. [ $\mathrm{e} n$ lín e a ], 1993. < Http://www. foodproductdesign. com/archive/1993/0493CS.html. >

14.HOGAM, S.A et al. Microencapsulation properties of whey protein concentrate 75 . Journal of food science. Vol. LXVI, (55), 2001, Pp. $675-680$

15.JACKSOM, L., LEE, K. Microencapsulation and the food industry. Lebensmittel Wissenschaft und Technologie. Vol. XXIV (4), 1991, PP. 289-297.

16.JAMDA, J., et al. Microencapsulation. US PATENT 5418010. 1995. Disponible en internet <http://www.uspto.gov>

17.LEVIME, H., et al. Double-encapsulated compositions containing volatile and/or labile components, and processes for preparation and use thereof. US PATENT 5087461. 1992. Disponible en internet http://www.uspto.gov

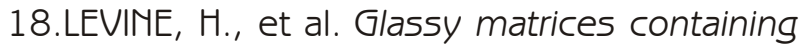
volatile and/or labile components, and processes for preparation and use thereof. US PATENT 5090900. 1991. Disponible en internet http://www.uspto.gov

19.MOREAU, D.L., ROSEMBERG, M. Porositiy of whey protein-based microcapsules containing anhydrous milkfat measured by Gas Displacement Picnometry. Journal of food science. Vol. LXIII (5), 1998, PP. 819-823.

20.ONWULATA, C.I. et al. Particle Structures of Encapsulated Milkfat Powders. Lebensmittel Wissenschaft und Technologie Vol. XXIX, 1996, pp.163-172.
21. OMWULATA, C.I., et al. Properties of singledouble-encapsulated butteroil powders. Journal of food science Vol. LXIII (1), 1998, pp.100 103.

22.PORZIO, M., POPPLEWEL, L. Encapsulation compositions. US PATENT 56039711997 Disp. en internet $<$ http://www.uspto.gov $>$

23.RÉ, M.I. Micorencapsulaçào em busca de produtos inteligentes. Ciencia hoie. Vol. XXVII (162), 2000, pp. 24-29.

24.REIMECCIUS, G.A. Carbohydrates for flavor encapsulation. Food Technology, Vol. XLIV (3), 1991, pp. 144-146.

25.SHAHIDI, F., HAM, X. Encapsulation of food ingredients. Critical reviews in Food Science and Mutrition, Vol. VI (33), 1993, Pp. 501-547.

26.SWISHER, H. Solid flavoring composition and method of preparing the same. US PATENT 2809895. 1957. Disponible en internet <http://www.uspto.gov>

27.VASISHTA, M. Microencapsulation: Delivering a market advantage. Prepared Foods. 2002. [en línea] <http://www.preparedfoods. Com/ archives/2002/2002_7/0702encap.htm>

28.VERSIC, R. Flavor encapsulation- an Overview. Disponible en internet <www.rtdodge.com/fl-ovrvw.htm >. Reimpreso de ACS Symposium Series Mo. 370, Flavor Encapsulation, Editores S.J. Risch and $\mathrm{G}$. A. Reinec 